\title{
Comparative Study of Unsteady Flows in a Transonic Centrifugal Compressor with Vaneless and Vaned Diffusers
}

\author{
Michael M. Cui \\ TRANE Air Conditioning, American Standard Companies, 3600 Pammel Creek Road, La Crosse, WI 54601, USA \\ Email: mcui@trane.com
}

Received 27 March 2003

To reduce vibration and noise level, the impeller and diffuser blade numbers inside an industrial compressor are typically chosen without common divisors. The shapes of volutes or collectors in these compressors are also not axis-symmetric. When impeller blades pass these asymmetric structures, the flow field in the compressor is time-dependent and three-dimensional. To obtain a fundamental physical understanding of these three-dimensional unsteady flow fields and assess their impact on the compressor performance, the flow field inside the compressors needs to be studied as a whole to include asymmetric and unsteady interaction between the compressor components. In the current study, a unified three-dimensional numerical model was built for a transonic centrifugal compressor including impeller, diffusers, and volute. HFC 134a was used as the working fluid. The thermodynamic and transport properties of the refrigerant gas were modeled by the Martin-Hou equation of state and power laws, respectively. The three-dimensional unsteady flow field was simulated with a Navier-Stokes solver using the $k-\varepsilon$ turbulent model. The overall performance parameters are obtained by integrating the field quantities. Both the unsteady flow field and the overall performance are analyzed comparatively for each component. The compressor was tested in a water chiller system instrumented to obtain both the overall performance data and local flow-field quantities. The experimental and numerical results agree well. The correlation between the overall compressor performance and local flow-field quantities is defined. The methodology developed and data obtained in these studies can be applied to the centrifugal compressor design and optimization.

Keywords and phrases: centrifugal compressor, impeller, diffuser, volute, unsteady flow, transonic flow.

\section{INTRODUCTION}

Adding vanes in a vaneless diffuser in a centrifugal compressor improves efficiency and reduces working range (Yoshinaga et al. [1], Flathers [2], Kmecl and Dalbert [3], and Cui [4]). Some local measurements suggest increased diffusion in vane channels (Yoshinaga et al. [1], Senoo et al. [5]). A more recent study (Cui [4]) indicates that the impact of the diffuser vanes spreads to all the components inside the compressor, including the impeller, diffuser, and volute. Cui analyzed the flow-field details at the steady state for the vaned and vaneless diffusers. The marked changes were found on the mass recirculation and pressure distortion near the volute tongue area as well as the pressure recovery along the volute centerline. The overall performance changes of compressors with vaned diffusers can be obtained by integrating changes in the

This is an open access article distributed under the Creative Commons Attribution License, which permits unrestricted use, distribution, and reproduction in any medium, provided the original work is properly cited. flow field caused by the diffuser vanes. Both local pressure distribution and overall pressure recovery coefficients from simulation agree well with measurements.

The current work extends the previous analysis to unsteady features of the flow field. Since the flow field inside the centrifugal compressor is inherently unsteady, the direct simulation of this unsteady flow will provide a fundamental physical understanding of the behavior of the compressor. The global performance characteristics can be obtained from integration of the local field quantities. The vibration and machinery sound of the compressor can also be characterized from these analyses.

To quantitatively evaluate the effects of vaned diffusers on compressor dynamic characteristics, the compressor configurations and operating conditions are kept the same for both diffusers. The only difference between the corresponding vaneless and vaned cases is that 13 vanes were added to the vaneless diffuser for the vaned diffuser case. The study was conducted in the perspective of compressor system and included both the overall performance and 


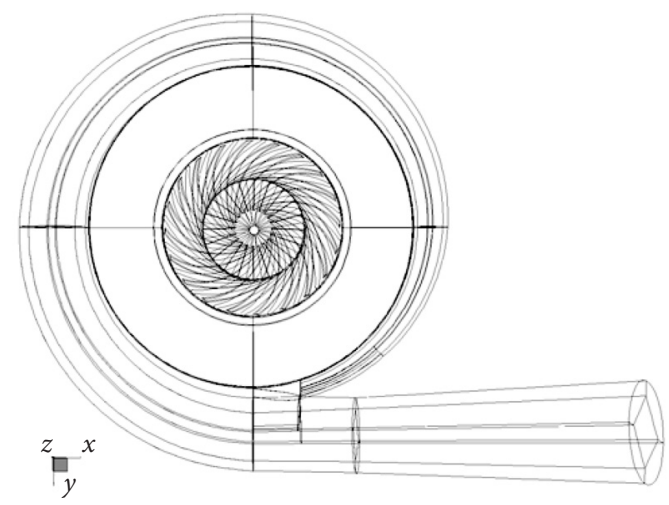

(a)

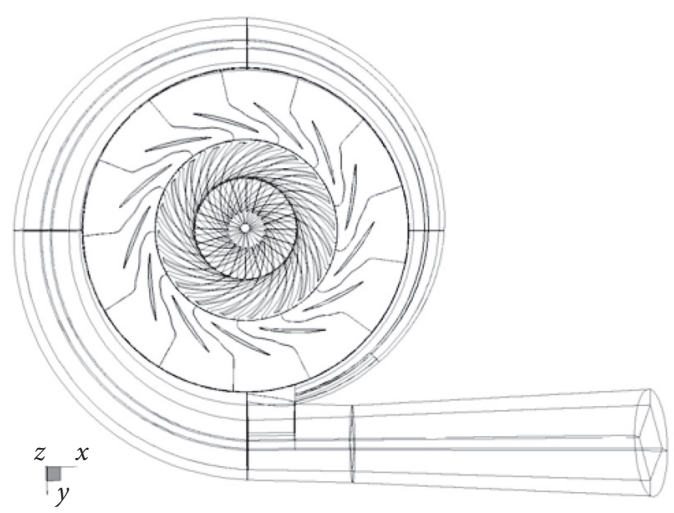

(b)

FIGURE 1: Numerical models of the compressors with the (a) vaneless and (b) vaned diffusers.

local flow-field details. The HFC-134a is the working fluid in this comparative study of the vaned and vaneless diffusers.

\section{METHODOLOGY}

The compressor, the testing facility, and data analysis procedures were described by Cui [4]. The structure of the numerical model was outlined in the same paper. For completeness, the block structures of the computational domain are included (Figure 1). The model is composed of 74 blocks and 690000 hexahedral elements. To simulate the real gas behavior near saturation, a user-defined program is applied to calculate the thermal and transport properties of HFC 134 a. The Navier-Stokes equations with the $k-\varepsilon$ turbulent model were solved with a coupled-implicit solver based on finite-volume method. The transient calculation uses the converged steady state solutions as its initial condition. The step length in the time domain used is one twentieth of the blade-passing period to ensure the resolution of the highfrequency component of the flow field. For each time step, the solution is considered to be converged when the RMS

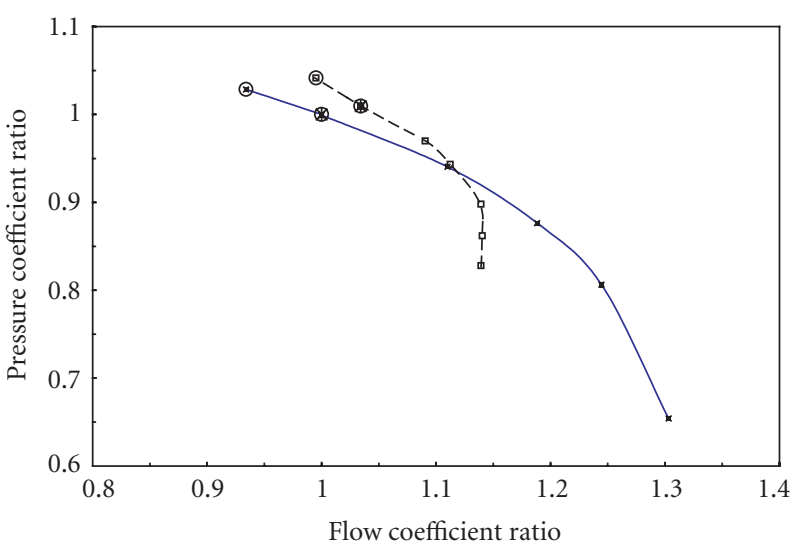

$$
\begin{array}{ll}
* \text { Vanelees diffuser } & * \text { Design points } \\
-*-\text { Vaned diffuser } & \circ \text { Calculation }
\end{array}
$$

Figure 2: Pressure coefficient of the compressor.

residuals of all of the governing equations have been reduced by five to six orders of magnitude from their initial values. The calculation continues until the main structure of the flow field shows no monotonic change of all field quantities.

A total of four cases are analyzed (Figure 2). Two of them are at the design conditions. The other two cases are at the near-surge conditions. The design conditions are dictated by the standard water chiller design conditions with $44^{\circ} \mathrm{F}$ of leaving-evaporator-water temperature and $85^{\circ} \mathrm{F}$ of enteringcondenser-water temperature. For the water chiller used in the current study, these design conditions provide the same inlet and exit pressure and temperature conditions. The nearsurge conditions were obtained by adjusting the entering condenser water temperature to reach the surge and then reducing the water temperature to obtain a surge-free operating point for the compressor.

Both instantaneous flow fields and statistical values of the physical quantities were obtained for each case analyzed. The length of time step is uniform during the simulation. From the viewpoint of statistics, the samples are uniformly distributed in the time domain. The mean value and variance of an arbitrary physical quantity, $\Phi$, that define its central tendency and dispersion, are estimated as follows:

$$
\begin{gathered}
\Phi=\frac{1}{N} \sum_{n=1}^{N} \Phi(n), \\
\Phi_{\mathrm{RMS}}=\left\{\frac{1}{N} \sum_{n=1}^{N}[\Phi(n)-\bar{\Phi}]^{2}\right\}^{1 / 2},
\end{gathered}
$$

where $\Phi(n)$ is the instantaneous value of $\Phi$ at time step $n$, and $N$ is the number of time steps used in the calculation.

The pressure level, $L_{p}$, is introduced to present the pressure variation inside the compressor. The same definition is also used in the analysis of variance of pressure, $P_{\mathrm{RMS}}$. The equations for computing pressure level and pressure 
fluctuation in $\mathrm{dB}$ are as follows:

$$
\begin{aligned}
L_{P} & =20 \log _{10} \frac{P}{P_{\text {ref }}}, \\
L_{\text {RMS }} & =20 \log _{10} \frac{P_{\text {RMS }}}{P_{\text {ref }}},
\end{aligned}
$$

where $P$ is the static pressure, $P_{\text {ref }}$ is a reference pressure value, and $P_{\mathrm{RMS}}$ is the variance of pressure.

The unsteady internal flow inside the compressor not only changes the compressor's performance but also leads to vibration and sound generation of the compressor and associated structure. The majority of this effect is through the force acting on the internal surface of the compressor. In the current study, the total force acting on the compressor resulting from the internal pressure and shear-stress distribution is calculated as follows:

$$
\vec{F}=\frac{\partial}{\partial t}\left(\oiiint_{\mathrm{CV}} \vec{V} \rho d v\right)+\oiint_{\mathrm{CS}} \vec{V} \rho(\vec{V} \cdot \vec{n}) d A
$$

where $A$ is area, CV is control volume, CS is control surface, $V$ is velocity, $t$ is time, $v$ is volume, and $\rho$ is fluid density.

The $x_{-}, y$-, and $z$-components of the total force, $F$, are calculated first. The magnitude of the total force acting on the internal surface of the compressor is then obtained by calculating the square root of the summation of the $x-, y$ and $z$-component squares:

$$
F=\sqrt{F_{x}^{2}+F_{y}^{2}+F_{z}^{2}}
$$

The digital fast Fourier transform is applied to the total force on the compressor to obtain the data in the frequency domain to evaluate the impact of unsteadiness of the flow field on the compressor and chiller structure. The data are also used to compare with the vibration and sound testing results.

The degree of unsteadiness is introduced to characterize the difference between the unsteadiness of different compressor designs. The vaneless diffuser case at design conditions is chosen as a reference case. For a new design, the percentage change of the variance of a physical quantity from the base case is the degree of the unsteadiness of the physical quantity. Since the variance of a physical quantity is also a field quantity, the volume average of the variance of the chosen physical quantity over the flow region is calculated first:

$$
\bar{\Phi}_{\mathrm{RMS}}=\frac{1}{v} \int \Phi_{\mathrm{RMS}} d v
$$

where $v$ is the volume of the flow region.

The degree of unsteadiness, $D$, is then calculated as follows:

$$
D=\frac{\left(\bar{\Phi}_{\mathrm{RMS}}\right)-\left(\bar{\Phi}_{\mathrm{RMS}}\right)_{\mathrm{ref}}}{\left(\bar{\Phi}_{\mathrm{RMS}}\right)_{\mathrm{ref}}} .
$$

The degree of unsteadiness can be calculated for the entire compressor or for each component. It can be used to distinguish one design from others quantitatively.

\section{OVERALL CHARACTERISTICS OF UNSTEADY FLOW}

The measured global performances of the compressor with vaned and vaneless diffusers are shown in Figure 2. The four cases analyzed are also marked on the curves. The measured pressure values are obtained by averaging three sets of test data. The analyzed results uses the pressure boundary conditions.

The unsteadiness of the flow field inside the compressor shows two distinctive mechanisms. The first mechanism is the jets from the impeller blade channels sweeping over the fixed structure and resulting in the flow-field fluctuation at blade-passing frequency. Since the impeller has 20 blades and rotates at $19000 \mathrm{rpm}$, the blade-passing frequency is $6333 \mathrm{~Hz}$. The second mechanism is induced by the volute tongue, which disturbs the flow field at impeller rotating frequency. The temporal variation of the pressure at the center of the diffuser vane channel inlet and outlet is shown in Figure 3. For the vaneless case, the data are obtained at the corresponding spatial locations, although there is no real vane channel. The vaneless case shows stronger fluctuation due to the structure of jets and wakes coming from the impeller. The larger difference between the pressure values at the inlet and outlet of the vaned diffuser indicates the better diffusion compared to the vaneless case. The waveform and amplitude of the pressure fluctuation in the vaned diffuser case are similar to what J. Kang and S. Kang [6] observed in their vane-island diffuser. To show the difference of the flow fields inside the compressor with the vaneless and vaned diffusers, the field quantities are studied during both periods at the same moments equally spaced over the periods.

The most obvious effect of the temporal variation of the flow field can be obtained from the vibration and machinery sound of the compressor. The internal flow-field fluctuation vibrates the compressor, setting the air around it in motion. The vibrations of the compressor were measured and plotted together with the calculated total force on the internal surface of the compressor (Figure 4). The spectra of vibrations and driving force are shown in the frequency domain. Although the transfer function of the compressor structure is unknown, the profile of vibration and the driving force on the compressor show a similar pattern in the frequency domain.

The mean and variance of the physical quantities are calculated over the entire flow field. The comparison of the calculated and measured mean pressure field is shown in Figures 5,6 , and 7 . The details of the diffuser design have been provided by Cui [4]. The flow angles at the diffuser inlets show both spatial and temporal variations. The static pressure is measured at 11 locations around the circumference at the inlets and outlets of the diffusers and impeller. Multiple 


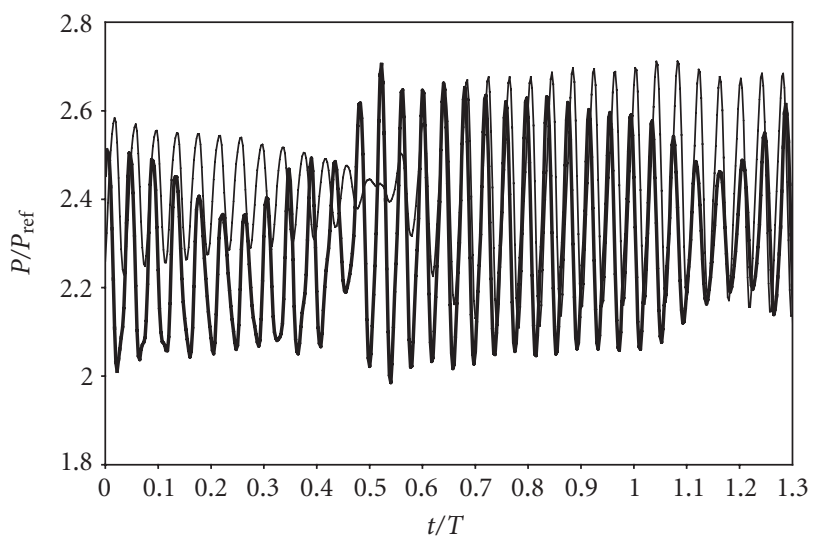

- Vane channel inlet near the volute tongue

- Vane channel outlet near the volute tongue

(a)

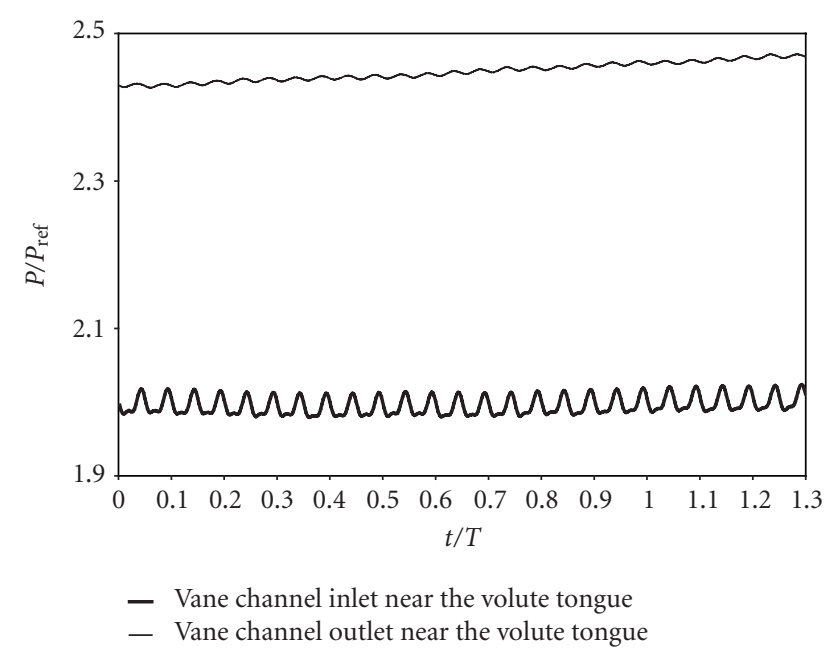

(b)

Figure 3: Temperal variation of the static pressure at the inlet and exit of the vane channel for the compressor with (a) vaneless and (b) vaned diffusers.

samples were taken over a period of time and simple algebraic average values were calculated. The locations of pressure measurement are the same for all the cases. The position of the volute tongue is also marked on these figures for reference. The mean value of pressure at the impeller inlet appears not to be effected by downstream structures. Since the averaged Mach number is close to unity, the perturbations from the diffuser and volute do not propagate upstream to the impeller inlet. This may not be the case for subsonic compressors (Hagelstein et al. [7]). In general, the results show that the time-averaged pressure value from transient simulation agrees well with the measurement. However, the timeaveraged pressure data show more deviation from the measurement in the vaned case compared to the vaneless case. The numbers of original data sets used in the statistical process for vaneless and vaned cases are 1154 and 521, respec-

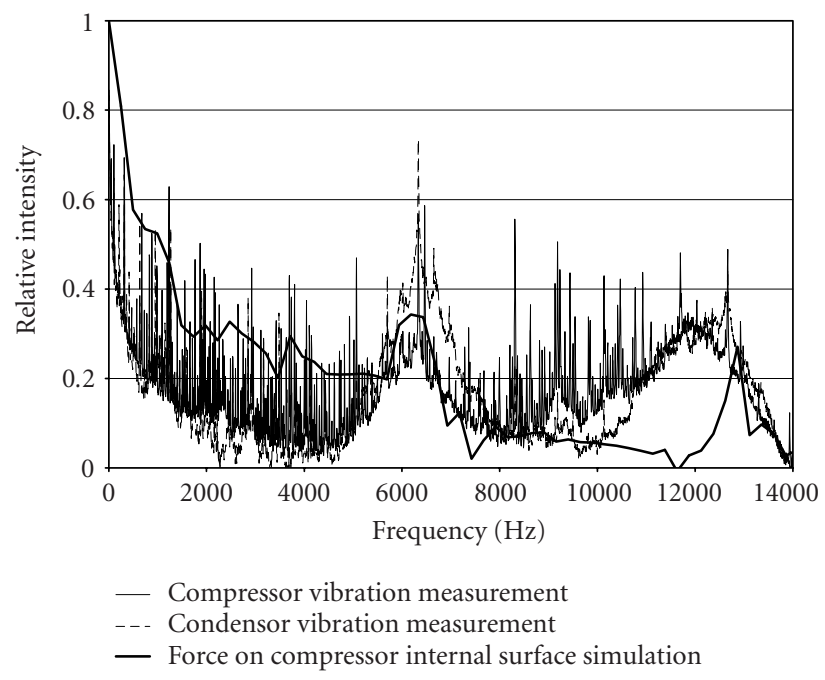

FIGURE 4: Spectra of compressor and condensor vibration and force on compressor internal surface for the vaned diffuser case at design conditions.

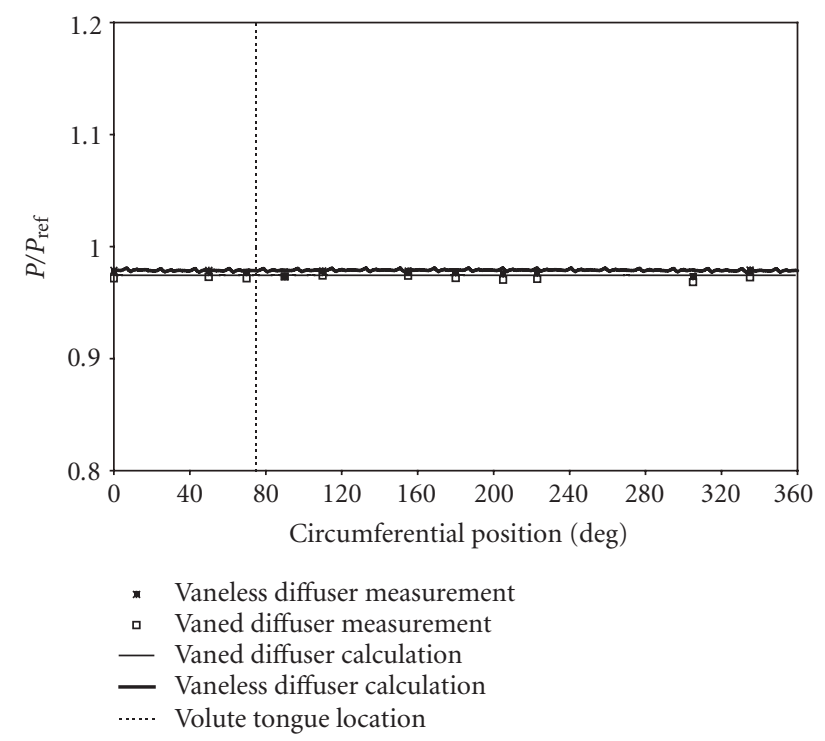

FIGURE 5: Static pressure at the inlet of the impeller for the vaneless and vaned diffusers.

tively. The statistical uncertainty may explain the difference of these results. A whole-field view of instantaneous velocity distribution can be see in Figure 8 . The $z$-component is plotted to show the different characteristics of the vaneless and vaned diffusers. The vaneless case shows a pattern of jets and wakes coming from the impeller. The vaned case indicates that the activities are concentrated near the diffuser inlet. Figures 9 and 10 show the fluctuation of the pressure field for both the vaneless and vaned cases at design conditions.

The degree of unsteadiness for the compressor with vaneless and vaned diffusers at design conditions is calculated using (6). Since the vaneless case is chosen as the reference 


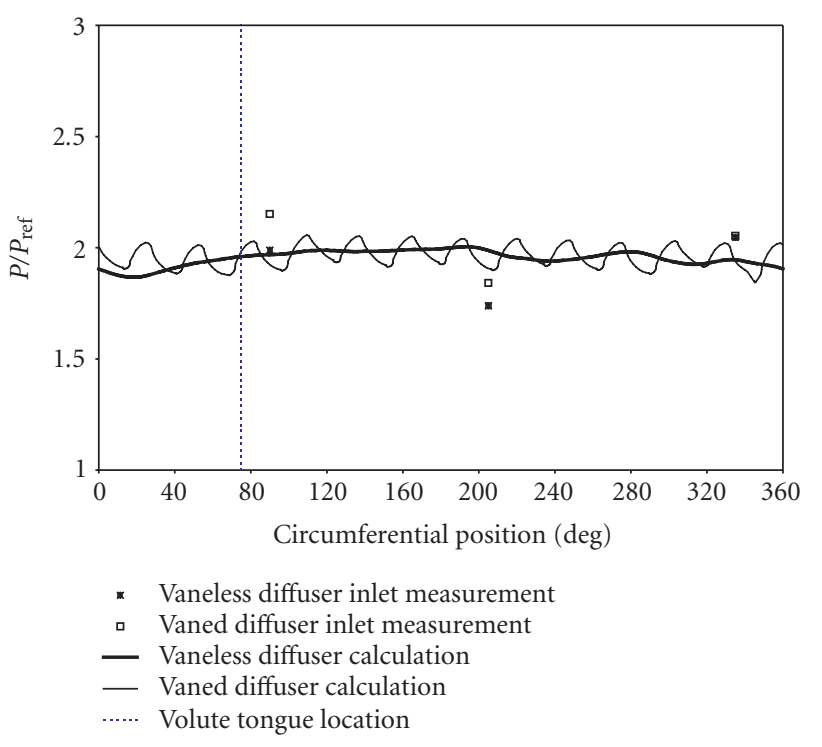

(a)

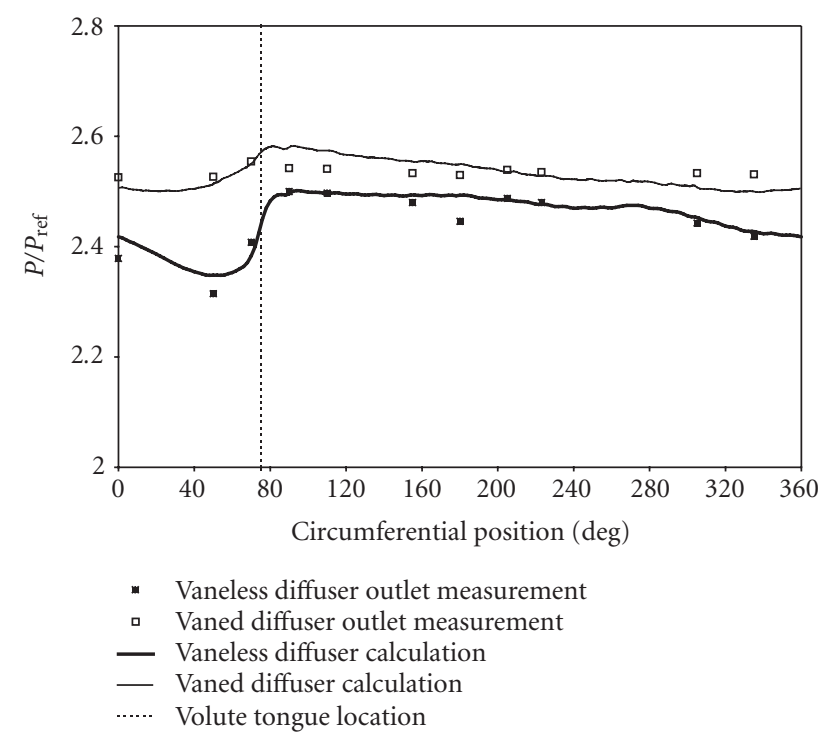

(b)

FIgURE 6: Static pressure at the (a) inlet and (b) outlet of the vaneless and vaned diffusers.

case, the degree of unsteadiness calculated for the vaned case indicates the difference between the two cases at design conditions. Table 1 shows a significant reduction $(-55 \%)$ in pressure fluctuation adding vanes to the diffuser. The lesser fluctuation leads to lesser mixing loss and turbulent dissipation inside the compressor. The calculated value of the degree of unsteadiness for the vaned case implies higher compressor efficiency. The measurements (Cui [4]) show an efficiency gain of $5 \%$. The significance of this comparison is that the unsteadiness needs to be studied not only at stall and surge conditions but also at design conditions.

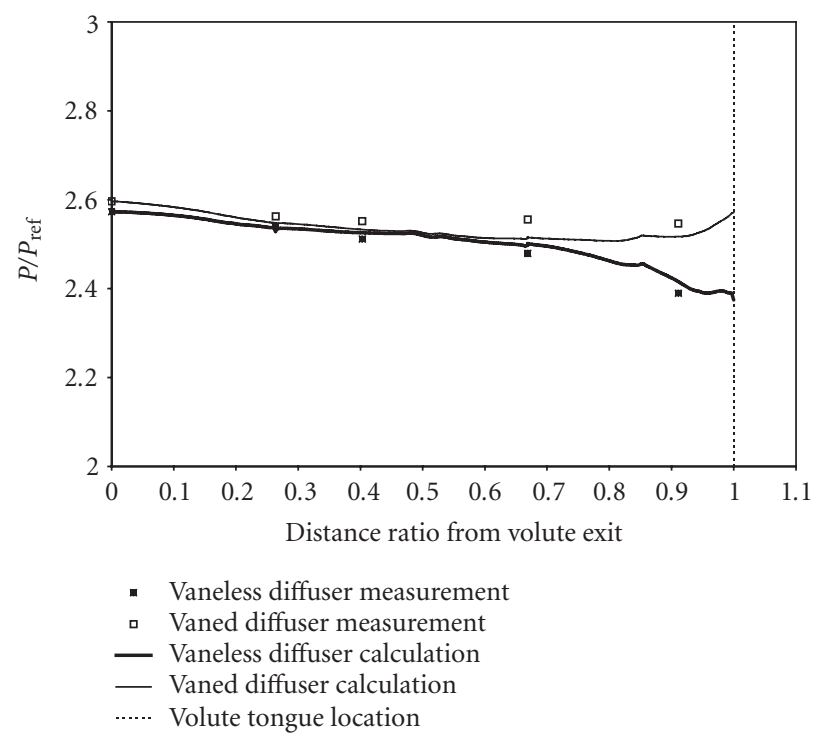

Figure 7: Static pressure on the volute wall for the vaneless and vaned diffusers.

TABLE 1: Unsteadiness degree of compressor.

\begin{tabular}{c|cc}
\hline \multirow{2}{*}{ Parameter } & \multicolumn{2}{|c}{ Compressor } \\
& Vanless & Vaned \\
\hline$D(\%)$ & 0 & -55.02 \\
\hline
\end{tabular}

During the compressor design and optimization, unsteadiness should be integrated into the process. The design with less flow fluctuation may have the potential to be more efficient, more reliable, and have less vibration and sound generation.

\section{IMPELLER FLOW}

The impeller pressure fields for the vaneless and vaned cases at design conditions are shown in Figures 11 and 12. The cases at near-surge conditions are shown in Figures 13 and 14, respectively. The vaneless case appears to have more variations around the impeller exit. The overall patterns of the pressure field for the vaneless case are asymmetric. The downstream asymmetric structure distorts the flow field inside the impeller. Hagelstein et al. [7] also observed the same phenomenon in their mean flow measurement. At the same design conditions, the vaned case shows an axis-symmetric pressure distribution independent of the impeller rotation. At the near-surge conditions (Figures 13 and 14), both vaneless and vaned cases show more variations of pressure at the impeller exit. The difference between the two cases is that these flow structures move along the circumference in the vaneless case and stay at the same spatial positions in the vaned case. The pressure field of the vaneless case shows a large degree of asymmetry. When the impeller rotates around the $z$-axis, the asymmetric impeller pressure field rotates at 


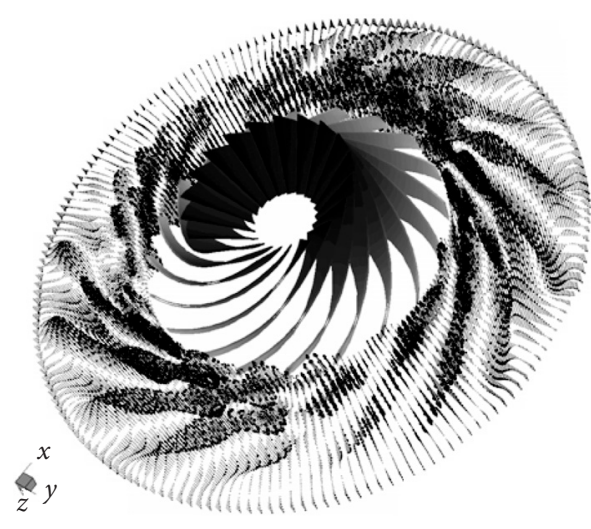

(a)

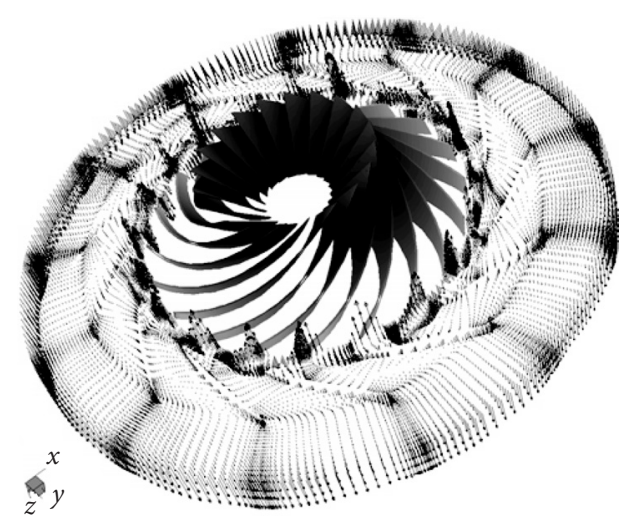

(b)

FIGURE 8: $z$-component of velocity on the diffuser center plane for the compressor with the (a) vaneless and (b) vaned diffusers.

a different speed (Figure 13) from the impeller rotating speed. The shapes of the isobaric surfaces of the vaneless case show a pattern of concentric circles and indicate that the vaneless case has a larger tangential velocity component. In the vaned case, the animated pressure field shows periodic oscillation near the exit along the impeller blade channels when the impeller blades pass the diffuser vanes. In the vaneless case, the volute tongue and mass recirculation around the tongue area effect the pressure field inside the impeller more. At the near-surge conditions, the difference between the vaneless and vaned cases widens. The vaneless case shows a near-elliptic pressure field rotating around the $z$-axis. The vaned case shows the impeller channels grouped together and are staying at the same spatial position in the absolute frame of reference (Figures 13 and 14).

The mean flow field obtained by averaging the instantaneous flow field shows that the diffuser vanes do not disturb the flow field at the impeller inlet (Figure 5). This result agrees with the steady-flow analysis and measurements (Cui [4]). However, the variance of the pressure shows strong effect of the diffuser vanes. An interesting phenomenon observed in the distributions of pressure variance (Figures

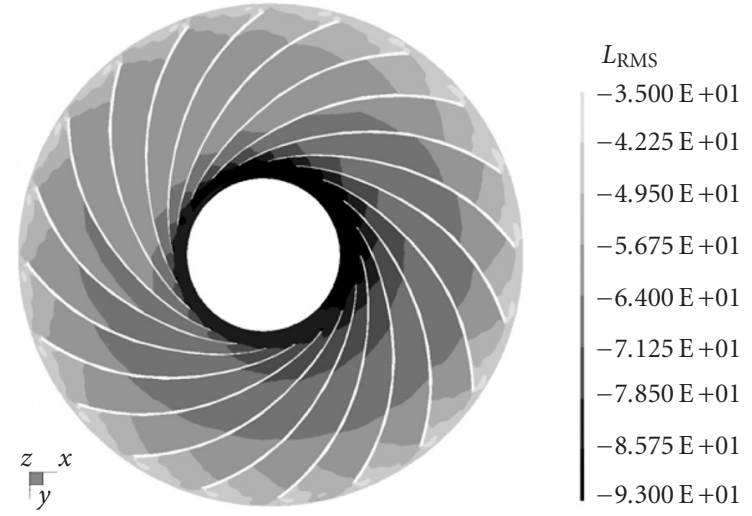

(a)

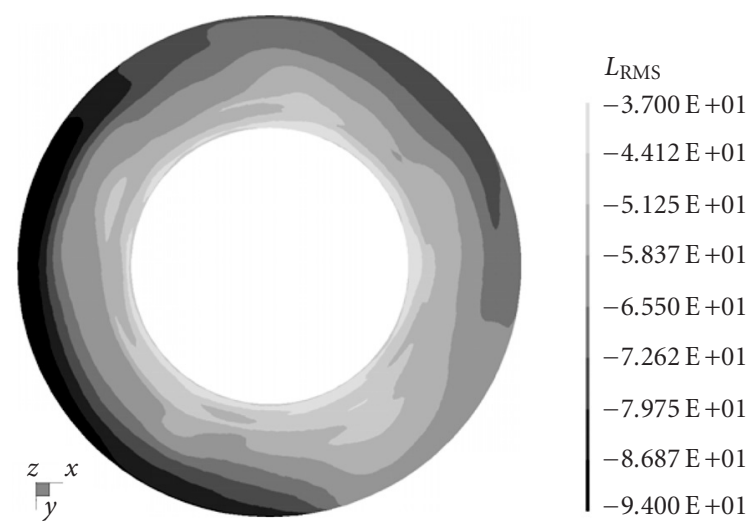

(b)

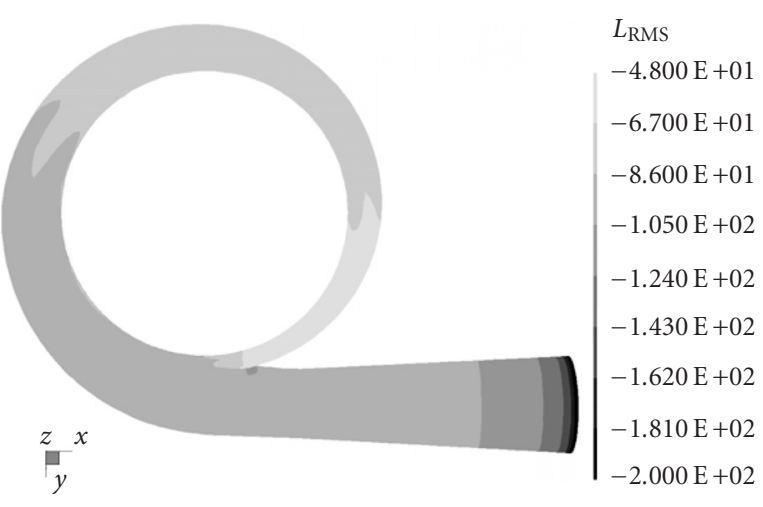

(c)

FIGURE 9: Variance of pressure level in the vaneless case at design conditions: (a) impeller center plane, (b) diffuser center plane, and (c) volute surface.

9 and 10) is that the pressure fluctuation in the vaneless case is asymmetric and its variation spreads over the entire impeller while the vaned case shows axis-symmetric pattern and variation limits to the impeller exit. The degree of the unsteadiness indicates a large difference between the two cases: the vanes added to the diffuser lead to an $82 \%$ 


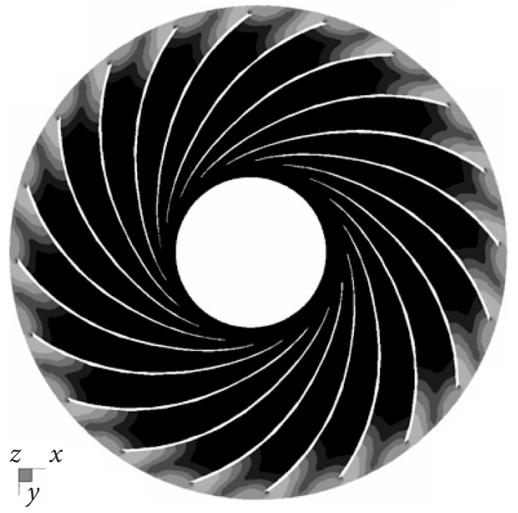

(a)

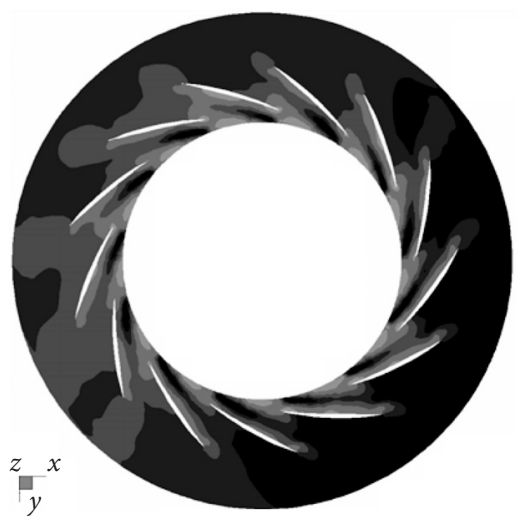

(b)

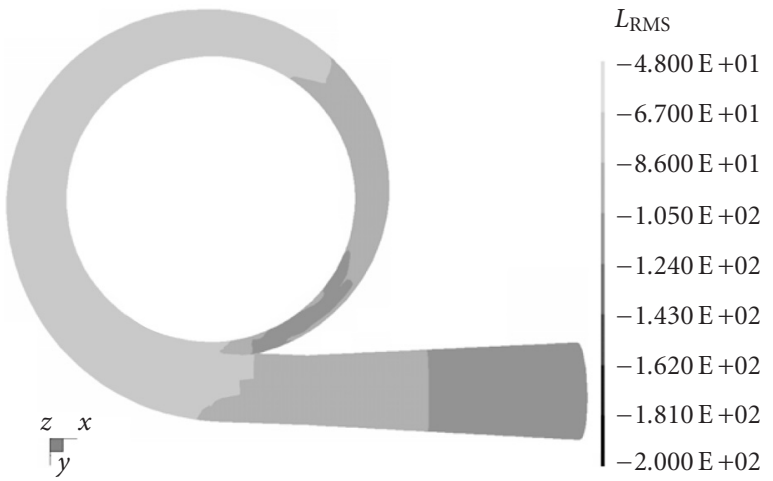

(c)

Figure 10: Variance of pressure level in the vaned case at design conditions: (a) impeller center plane, (b) diffuser center plane, and (c) volute surface.

TABLE 2: Unsteadiness degree of impeller.

\begin{tabular}{c|cc}
\hline \multirow{2}{*}{ Parameter } & \multicolumn{2}{|c}{ Impeller } \\
& Vanless & Vaned \\
\hline$D(\%)$ & 0 & -82.27 \\
\hline
\end{tabular}

reduction in unsteadiness. Again, the degree of unsteadiness points to the same direction as the compressor efficiency (see Table 2).

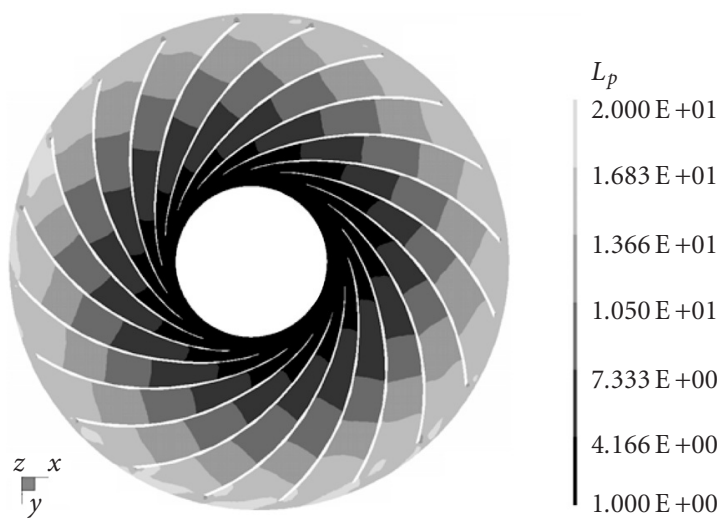

(a)

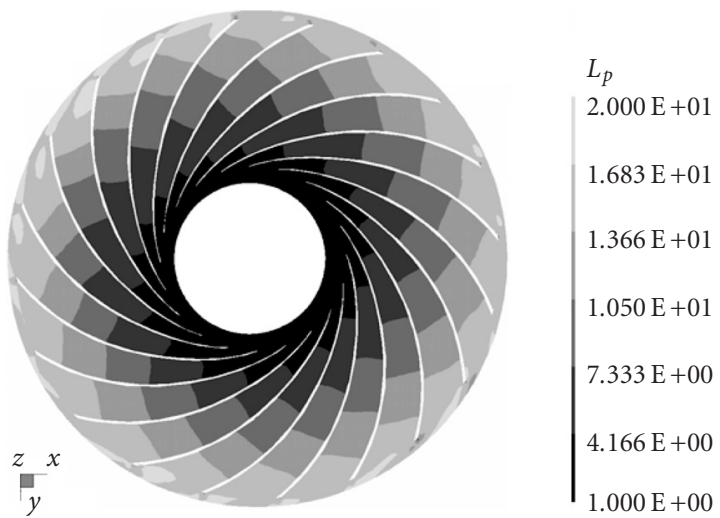

(b)

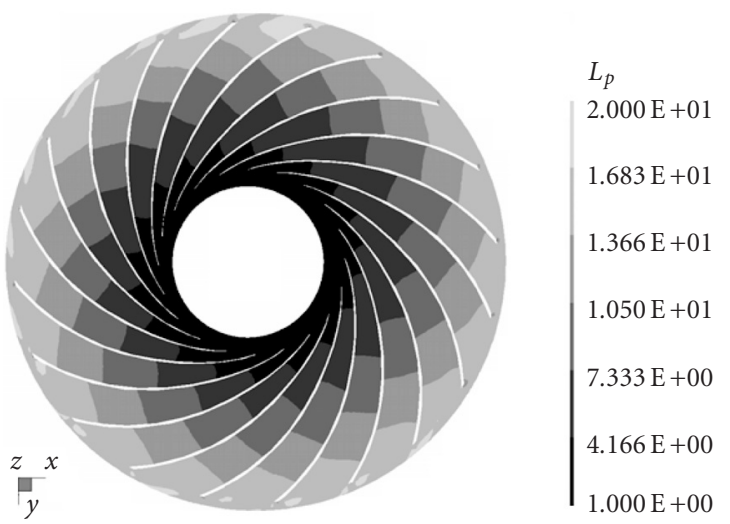

(c)

FIGURE 11: Static pressure distribution on impeller center plane in the vaneless diffuser at design conditions: (a) starting position, (b) $1 / 3$ revolution, and (c) $2 / 3$ revolution.

\section{DIFFUSER FLOW}

The static pressure distributions inside the vaneless and vaned diffusers at design conditions are shown in Figures 15 and 16, respectively. The diffuser flow is controlled by different physical mechanisms for the vaneless and vaned diffusers. Wakes and jets coming from the impeller dominate the flow inside the vaneless diffuser. The stripe-like pattern stretches along the flow direction leaving the impeller. These results 


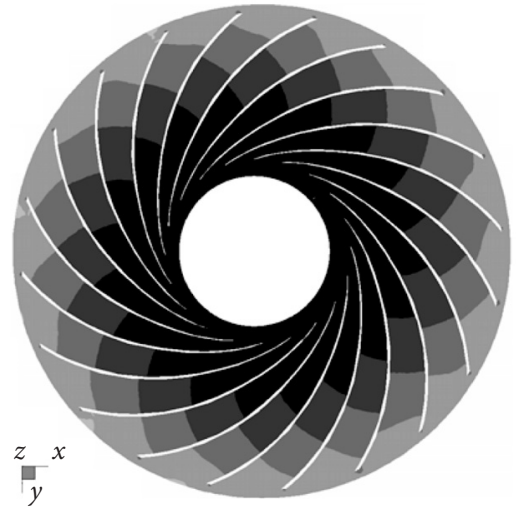

(a)

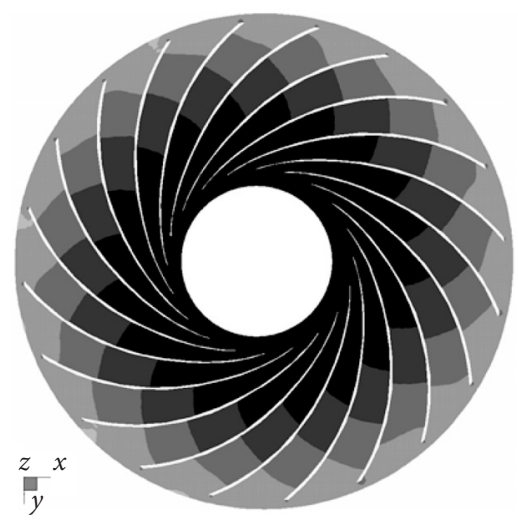

(b)

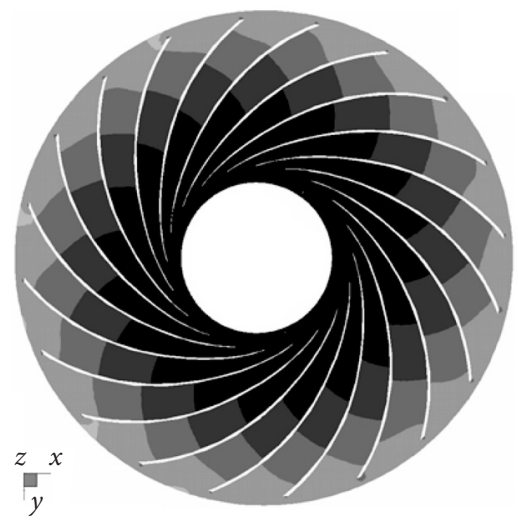

(c)

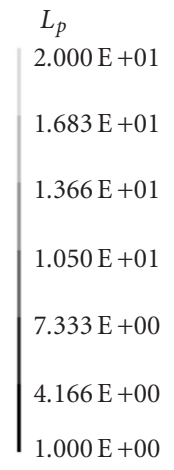

$L_{p}$

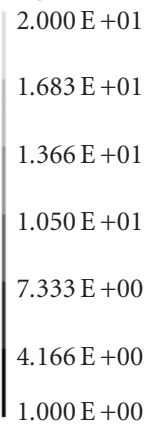

$1.000 \mathrm{E}+00$

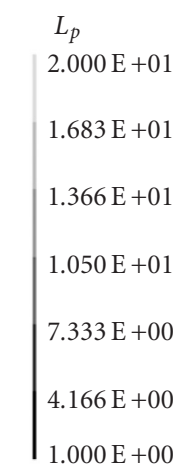

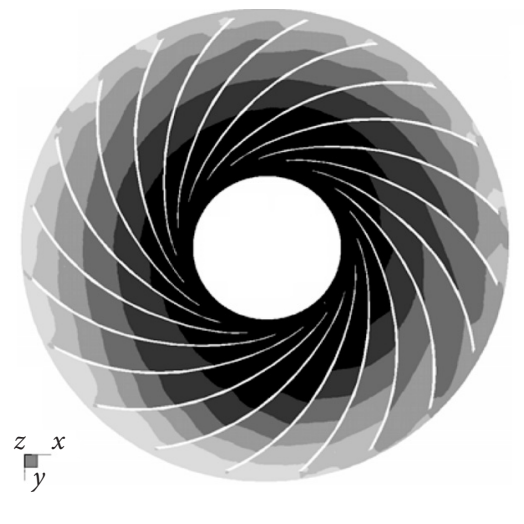

$L_{p}$

$2.000 \mathrm{E}+01$

$1.683 \mathrm{E}+01$

$1.366 \mathrm{E}+01$

$1.050 \mathrm{E}+01$

$7.333 \mathrm{E}+00$

$4.166 \mathrm{E}+00$

(a)

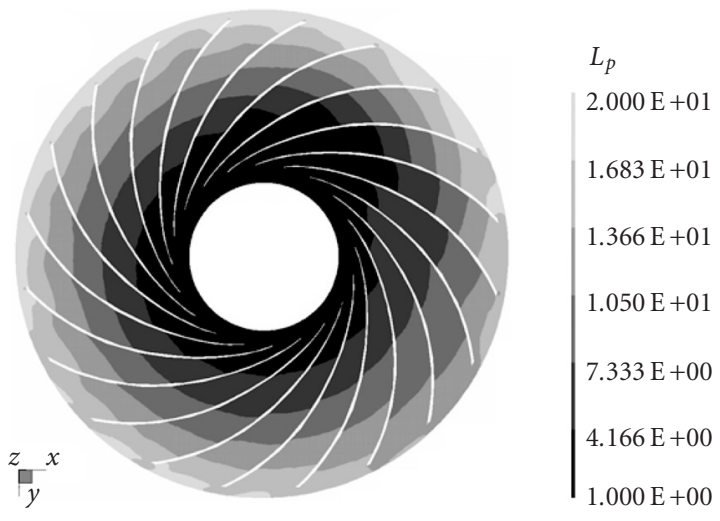

(b)

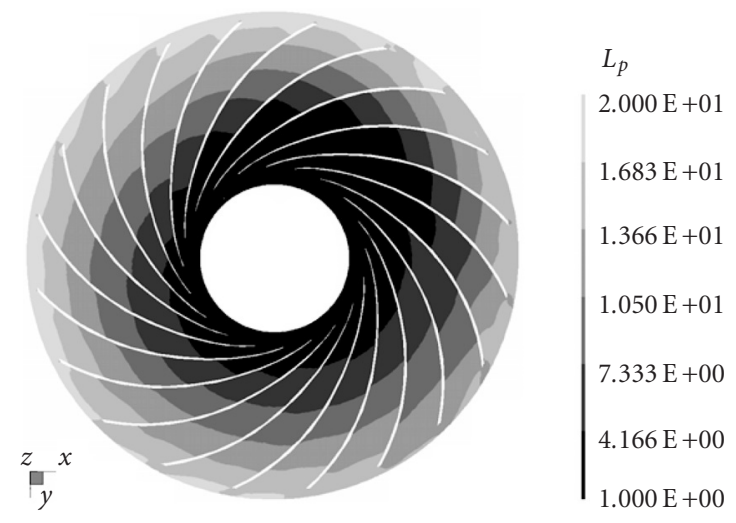

(c)
FIGURE 12: Static pressure distribution on impeller center plane in the vaned diffuser at design conditions: (a) starting position, (b) $1 / 3$ revolution, and (c) $2 / 3$ revolution.

agree with the observation of Roduner et al. [8]. The volute tongue has a strong impact on the overall pattern of the pressure field. When the impeller rotates around the $z$-axis, the pressure field inside the vaneless diffuser shows a large degree of variation. The stripes formed by the jets and wakes
FIGURE 13: Static pressure distribution on impeller center plane in the vaneless case at near-surge conditions: (a) starting position, (b) $1 / 3$ revolution, and (c) $2 / 3$ revolution.

rotates with the impeller at the same speed. The influence of the volute tongue is clearly observable. The vaned diffuser shows strong influence of the diffuser vanes. The pressure distribution at the diffuser inlet shows a series of standing waves with 13 peaks corresponding to 13 diffuser vanes. 

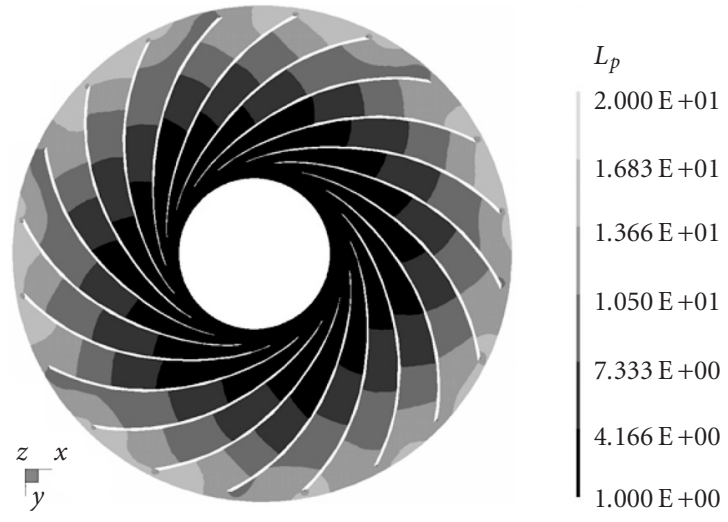

(a)

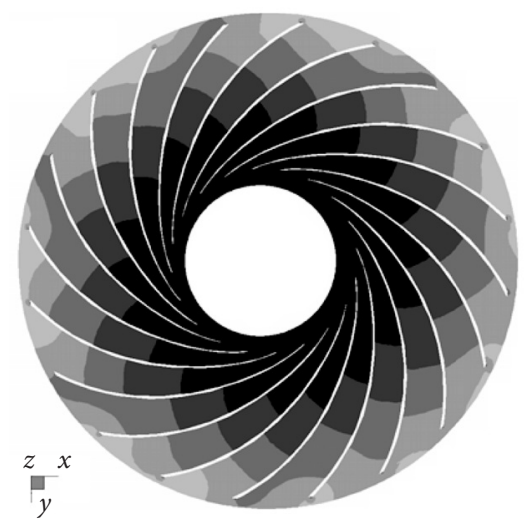

(b)
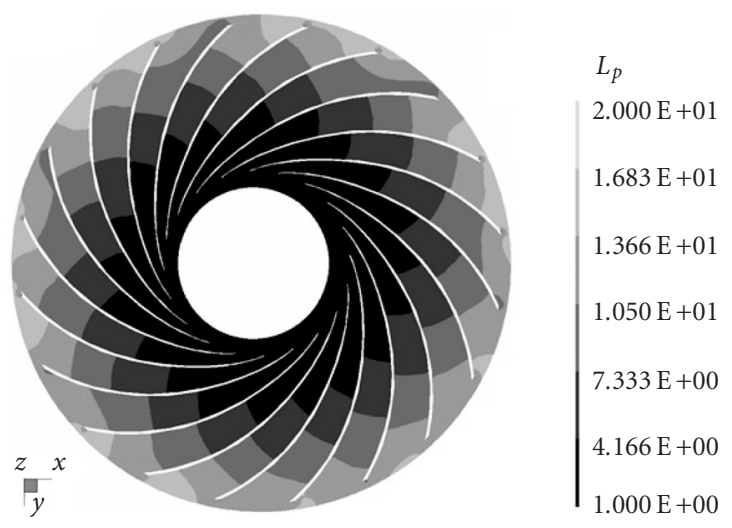

(c)

FIGURE 14: Static pressure distribution on impeller center plane in the vaned case at near-surge conditions: (a) starting position, (b) $1 / 3$ revolution, and (c) $2 / 3$ revolution.

The influence of the volute tongue is much weaker compared to the vaneless cases. When the impeller rotates, the standing waves corresponding to the diffuser vanes keep their circumferential position and only change their shapes and amplitudes. The 13 standing waves have different sizes. Pressure animation shows that the smaller waves are struggling to survive. This observation seems to support the practice

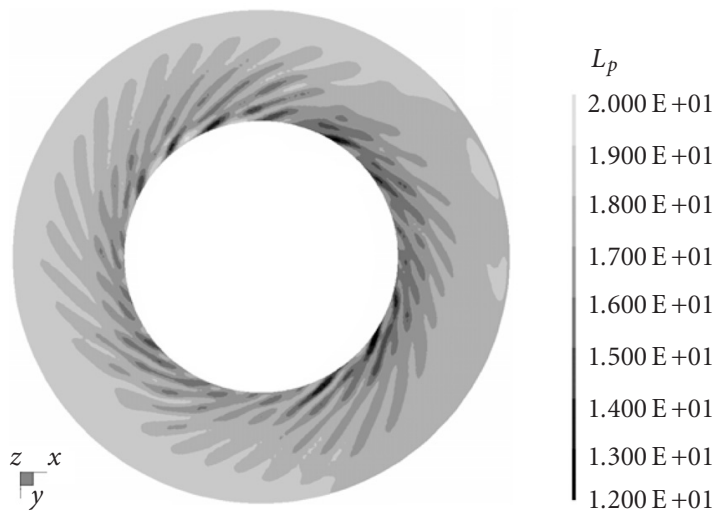

(a)

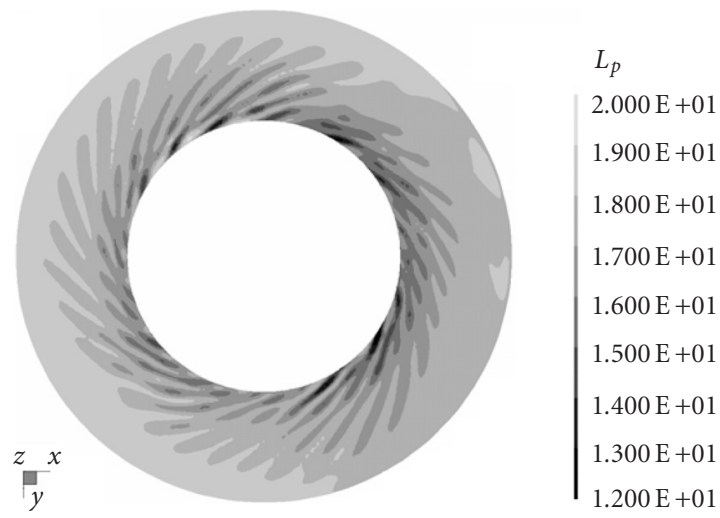

(b)

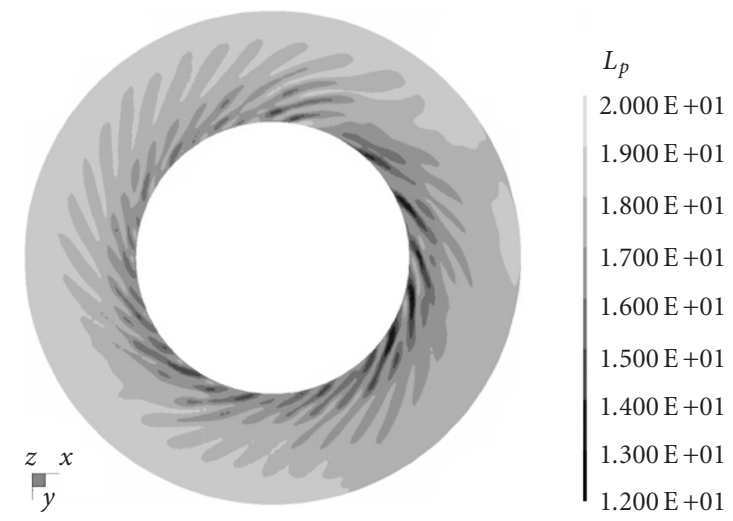

(c)

Figure 15: Static pressure on the vaneless diffuser center plane at design conditions: (a) starting position, (b) 1/3 revolution, and (c) $2 / 3$ revolution.

to choose the numbers of impeller blades and diffuser vanes without common divisors. The combination of 20 impeller blades and 13 diffuser vanes prevents the formation of 13 strong standing waves.

At the near-surge conditions shown in Figures 17 and 18 , the difference between the vaned and vaneless cases grows. The vaneless case shows that large structures are 


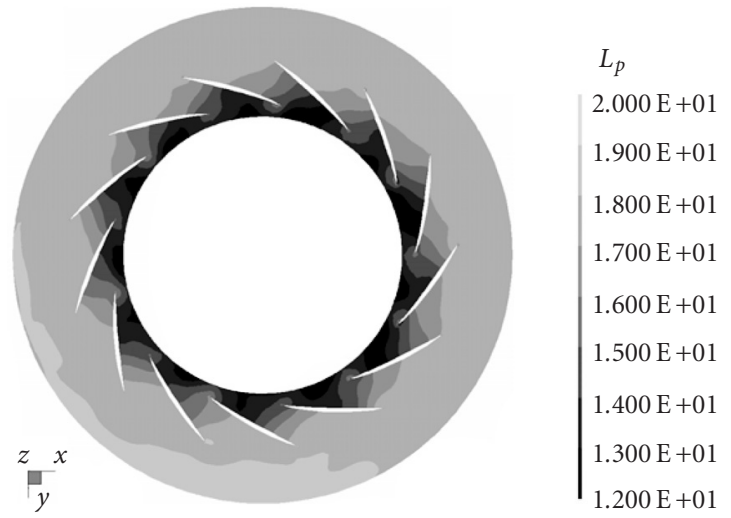

(a)

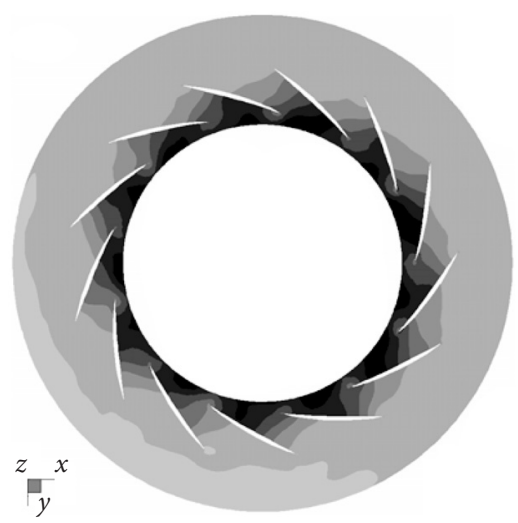

(b)

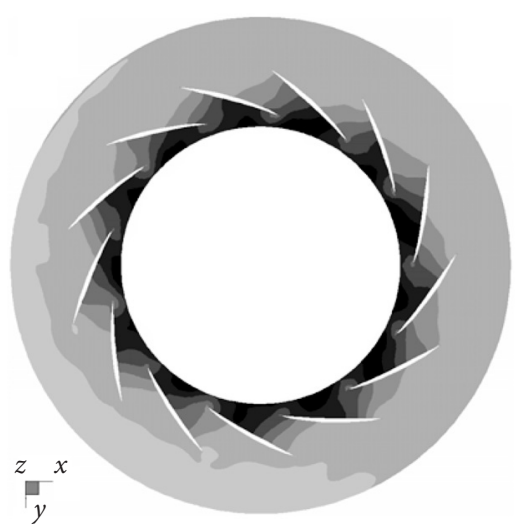

$L_{p}$
$2.000 \mathrm{E}+01$
$1.900 \mathrm{E}+01$
$1.800 \mathrm{E}+01$
$1.700 \mathrm{E}+01$
$1.600 \mathrm{E}+01$
$1.500 \mathrm{E}+01$
$1.400 \mathrm{E}+01$
$1.300 \mathrm{E}+01$
$1.200 \mathrm{E}+01$

(c)

FIGURE 16: Static pressure on the vaned diffuser center plane at design conditions: (a) starting position, (b) $1 / 3$ revolution, and (c) 2/3 revolution.

continuously generated from the impeller and span off with lower frequency than the impeller rotating speed. The stripes in the pressure field generated by jets and wakes are no longer dominating while the impact of the volute tongue is more obvious. The vaned diffuser case still shows standing waves at the inlet. An interesting phenomenon is that the number of

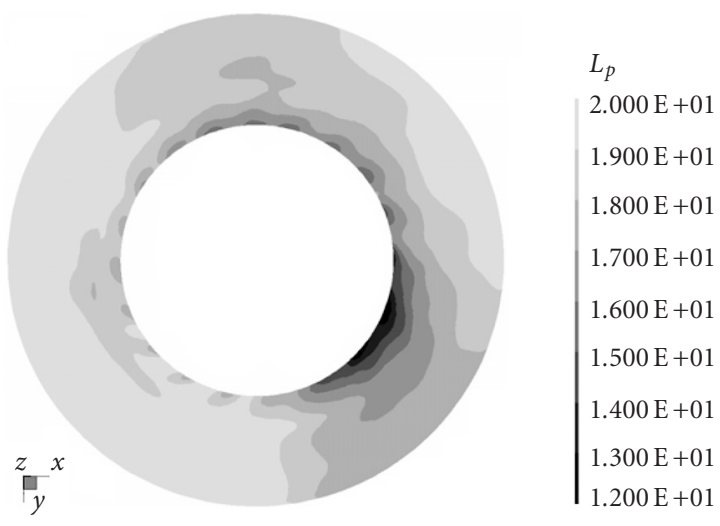

(a)

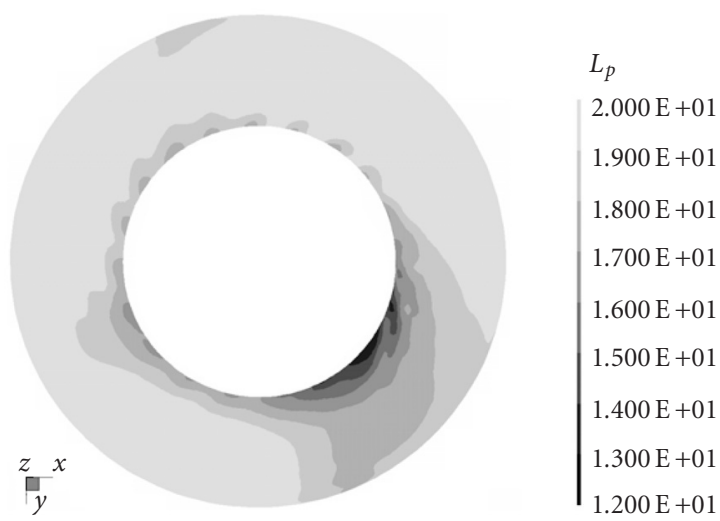

(b)

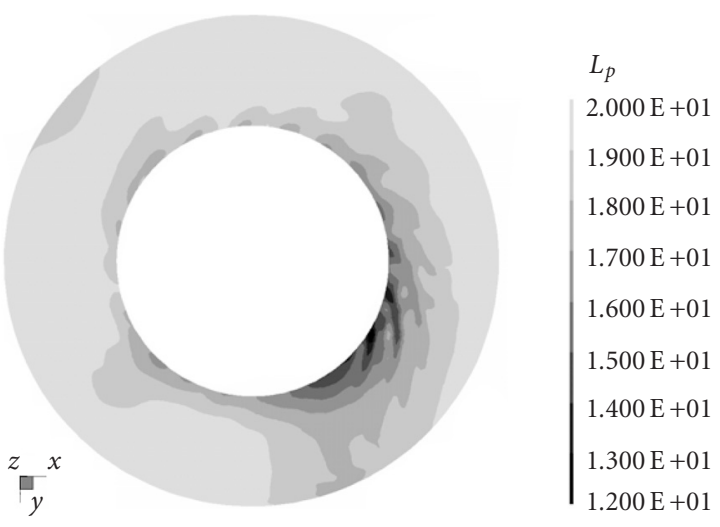

(c)

Figure 17: Static pressure on the diffuser center plane for the vaneless case at near-surge conditions: (a) starting position, (b) 1/3 revolution, and (c) $2 / 3$ revolution.

the standing waves changes from 13 at the design conditions to eight at the near-surge conditions. Among the eight standing waves, six of them are much stronger than two smaller ones. The pressure field intends to form standing waves with the wave number being the multiple of the common divisors of the impeller and diffuser vane numbers. The pressure field 


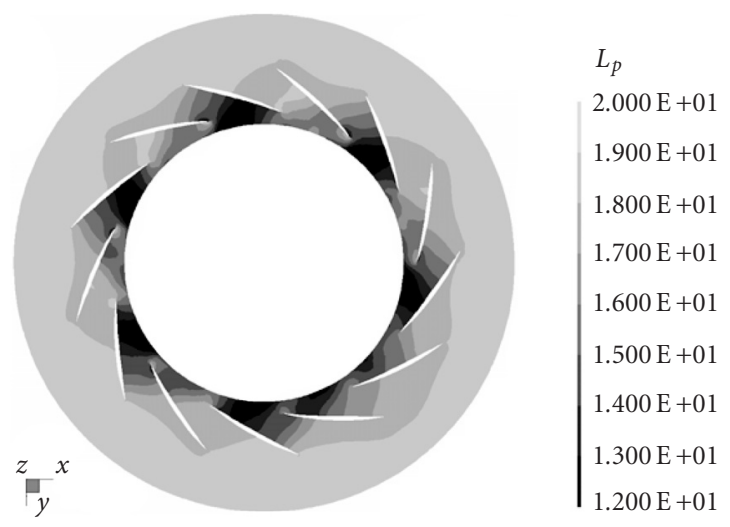

(a)

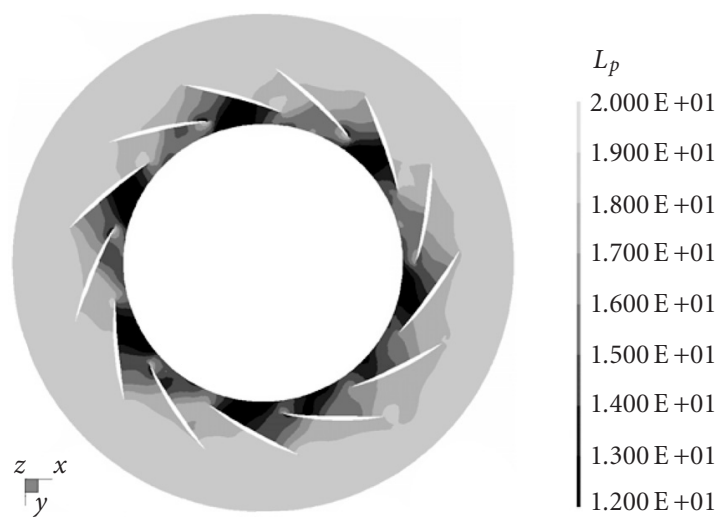

(b)

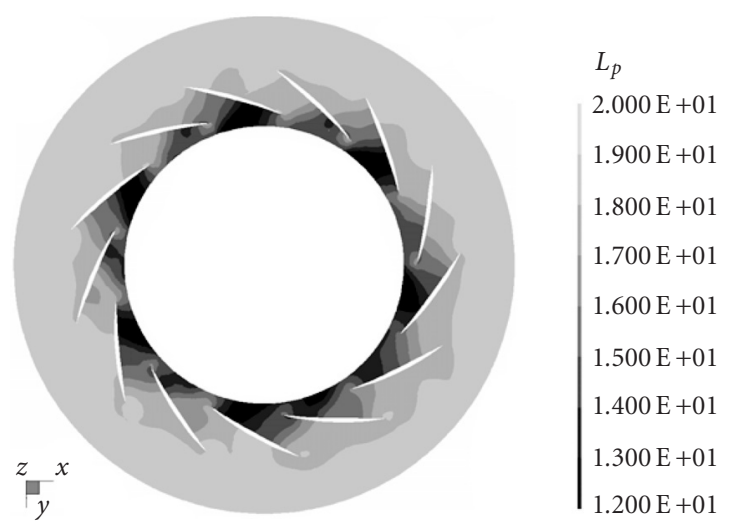

(c)

FIGURE 18: Static pressure on the diffuser center plane for the vaned case at near-surge conditions: (a) starting position, (b) 1/3 revolution, and (c) $2 / 3$ revolution.

animation shows that the pressure along the vane channel oscillates periodically. When the vaneless case shows stronger volute tongue influence, the vaned case shows very limited impact from the volute tongue.

The variance of the pressure field in the vaneless and vaned diffusers is shown in Figures $9 \mathrm{~b}$ and $10 \mathrm{~b}$. The pressure
TABle 3: Unsteadiness degree of diffuser.

\begin{tabular}{c|cc}
\hline Parameter & \multicolumn{2}{|c}{ Diffuser } \\
\hline$D(\%)$ & Vanless & Vaned \\
\hline
\end{tabular}

TABLE 4: Unsteadiness degree of volute.

\begin{tabular}{c|cc}
\hline \multirow{2}{*}{ Parameter } & \multicolumn{2}{|c}{ Volute } \\
& Vanless & Vaned \\
\hline$D(\%)$ & 0 & -1.13 \\
\hline
\end{tabular}

inside the vaneless diffuser fluctuates significantly more than inside its vaned counterpart. The pressure fluctuation is more concentrated inside the diffuser vane channels in the vaned case while in the vaneless diffuser it spreads widely over the entire diffuser. The volute tongue effects the pressure fluctuation for both the vaned and vaneless cases. In the vaned case, the variance of pressure distribution is more asymmetric compared to the mean value distribution of the pressure. The diffuser vanes contribute significantly to the reduction of the degree of unsteadiness of the vaned diffuser. The degree of unsteadiness decreased more than 90\% compared to the vaneless case (see Table 3 ).

\section{VOLUTE FLOW}

The pressure field variation at design conditions inside the volute is shown in Figures 19 and 20. The vaneless case still shows more variation along the circumference and changes more when the impeller rotates compared to the vaned diffuser case. However, the range of variation in the volute is smaller than that in the impeller and diffuser.

At near-surge conditions, the vaneless case shows large flow structures moving down the flow passage and a significant pressure variation inside the volute (Figures 21 and 22). These large structures are continuously generated by the impeller and move down to the diffuser and volute. Although these structures are moving at different tangential speeds than the impeller, they originally gain their momentum from the impeller motion and move in the mean flow direction. In the vaned diffuser case, the pressure inside the volute is rather stable and independent of the impeller rotation. While the large structures continuously move from the impeller down to the volute in the vaneless case, there is no significant temporal variation around the volute circumference in the vaned case. The unsteadiness in the vaned case apparently concentrates near the diffuser vanes. The oscillations occur along the vane channels and show a stable spatial pattern. The volute tongue and recirculation in the tongue area make the pressure reaching its lowest value near the tongue region of the volute for both vaneless and vaned cases.

The pressure fluctuation is less intensive compared to the impeller and diffuser at the design conditions for both the vaneless and vaned cases (Figures 9 and 10). As the result, the degree of unsteadiness calculated from the variance of the pressure field is also lower (Table 4). However, there is 


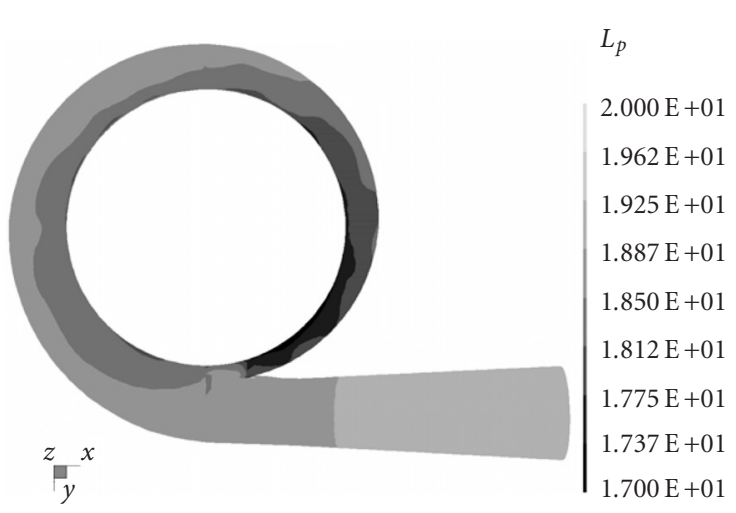

(a)

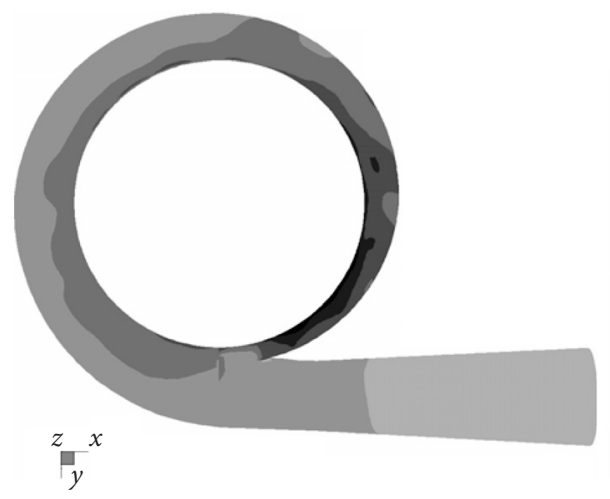

$L_{p}$

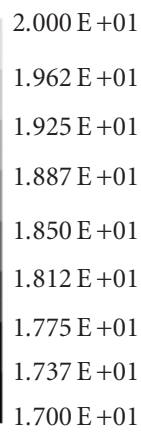

(b)

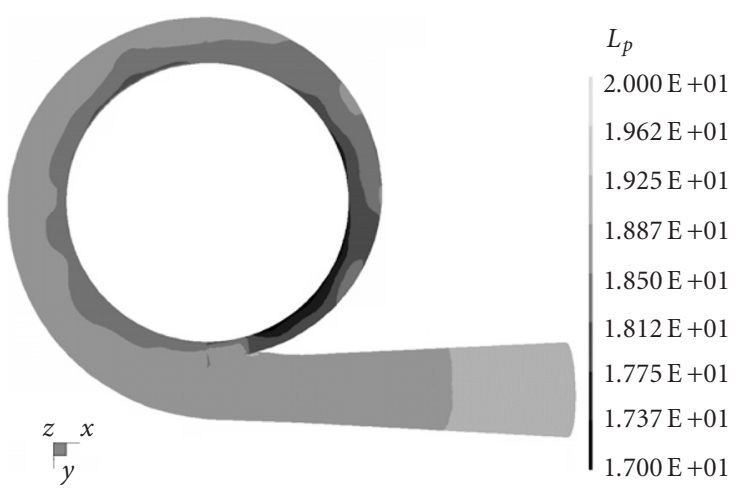

(c)

FIGURE 19: Static pressure on the volute surface for the vaneless case at design conditions: (a) starting position, (b) $1 / 3$ revolution, and (c) $2 / 3$ revolution.

a difference between the two cases: the vaneless case shows more fluctuation near the volute tongue while the vaned case is more stable in the same region. The same feature can be observed in the pressure fluctuation pattern in the vaned diffuser.

\section{CONCLUSIONS}

Adding vanes in the vaneless diffuser causes significant change in the unsteady flow field inside the compressor.

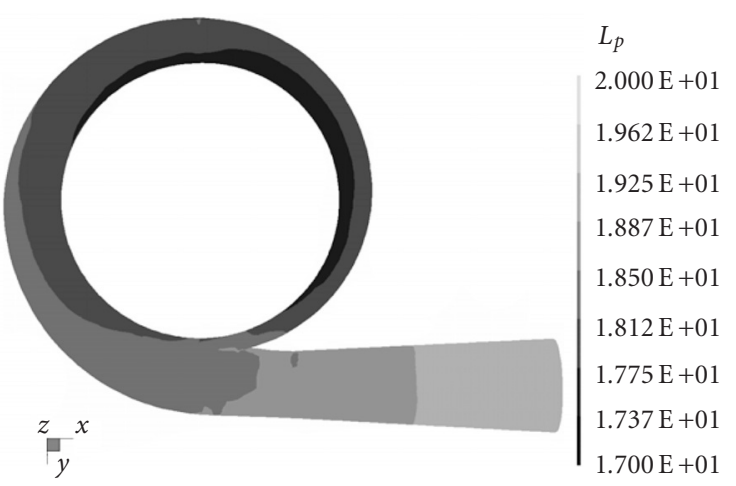

(a)

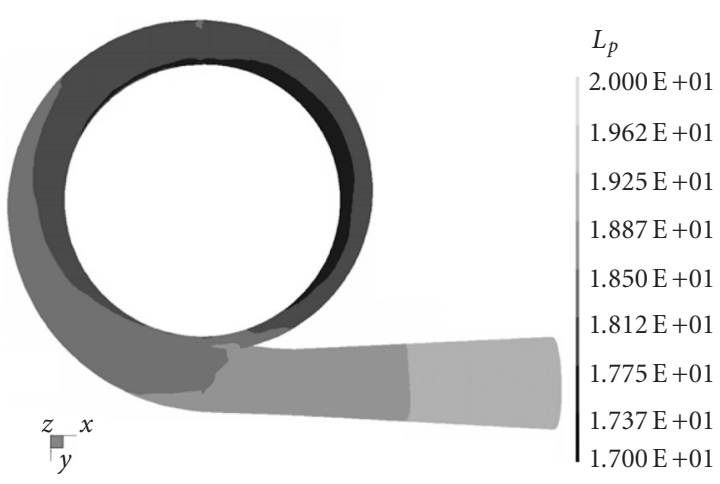

(b)

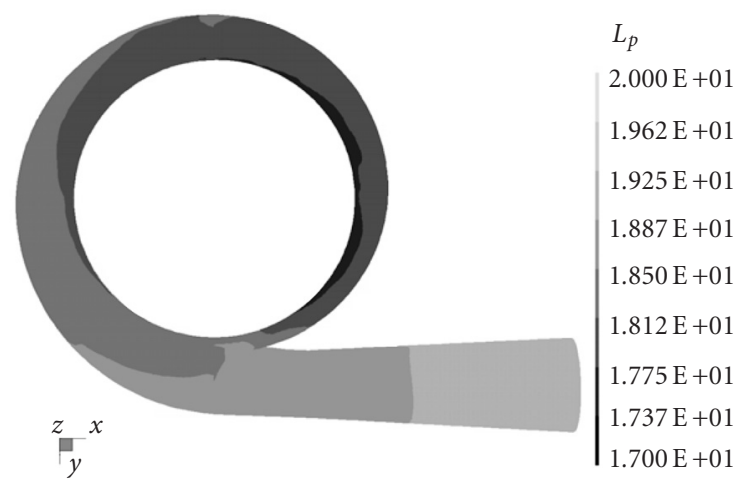

(c)

FIGURE 20: Static pressure on the volute surface for the vaned case at design conditions: (a) starting position, (b) $1 / 3$ revolution, and (c) $2 / 3$ revolution.

These changes spread over the entire flow field and result in the different overall performance for the same compressor. In general, the degree of the unsteadiness in the compressor is reduced. The reduction of the unsteadiness naturally leads to less mixing loss and turbulent energy dissipation in the system. This mechanism provides an explanation of higher compressor efficiency.

The unsteady flow analysis indicates that the flow fluctuation pattern is changed in addition to the mean flow field. Unlike the widely spreading flow fluctuation in the vaneless 


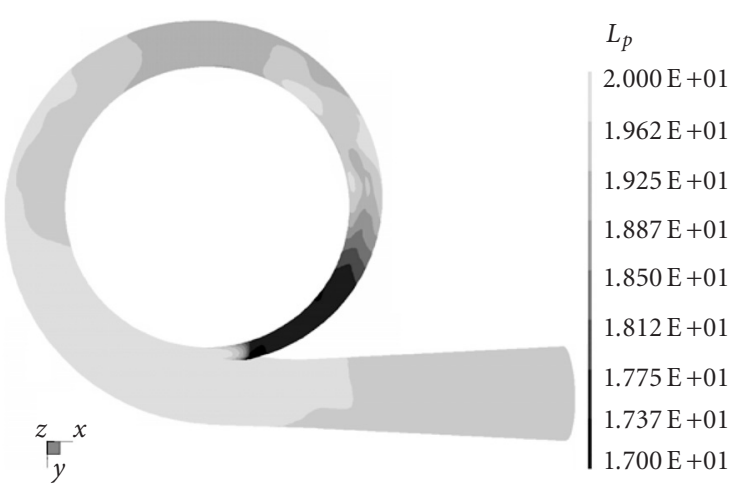

(a)

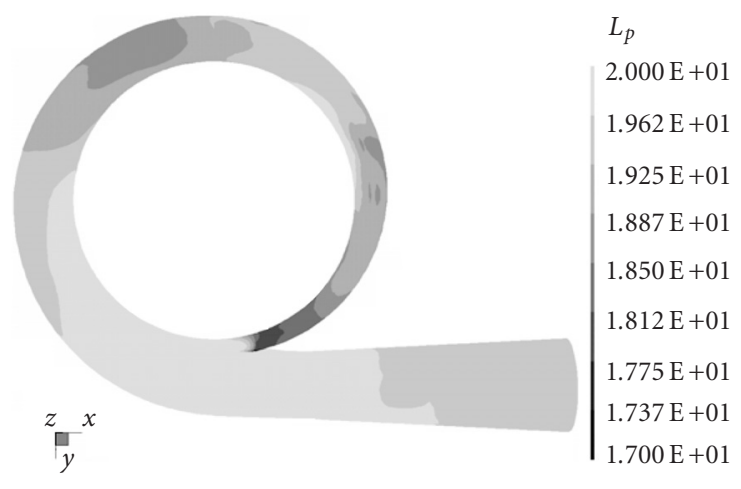

(b)

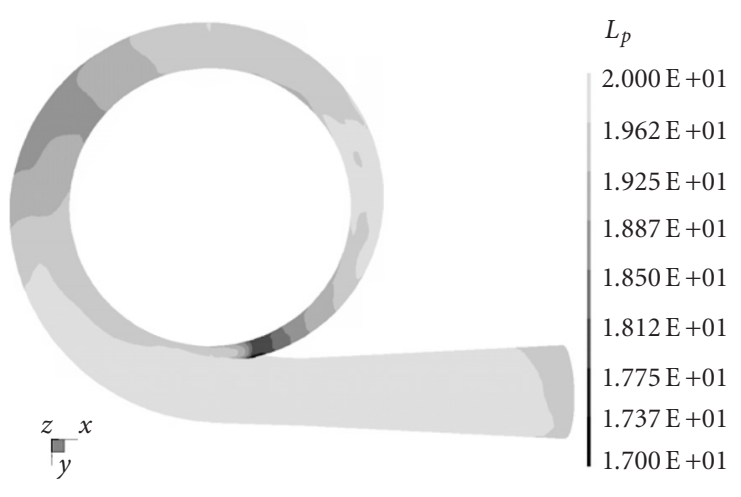

(c)

FIGURE 21: Static pressure on the volute surface for the vaneless case at near-surge conditions: (a) starting position, (b) 1/3 revolution, and (c) $2 / 3$ revolution.

case, the flow in the vaned case shows a more controlled behavior. The temporal variation of the field quantities concentrates on the region near the impeller exit and diffuser vane channels. The information about this localization provides a direction for future improvement in the compressor performance and design optimization. The right design should reduce the unsteadiness in these regions and improve the

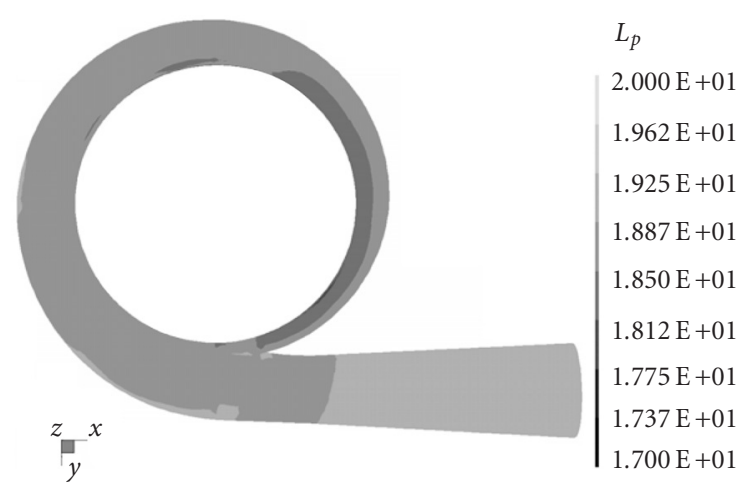

(a)

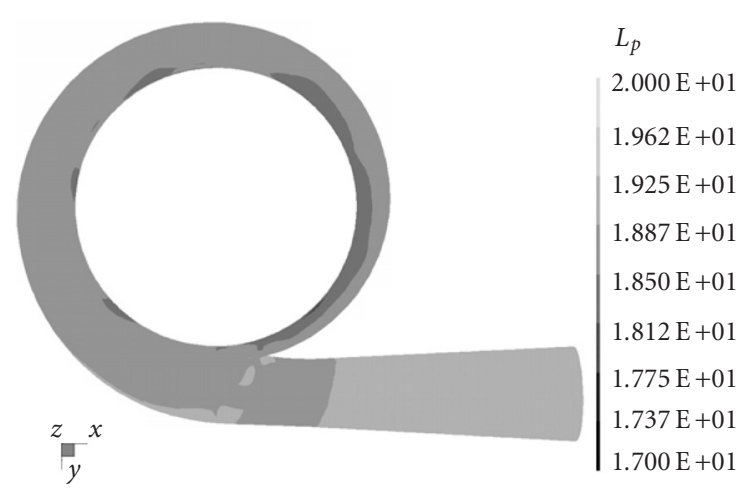

(b)

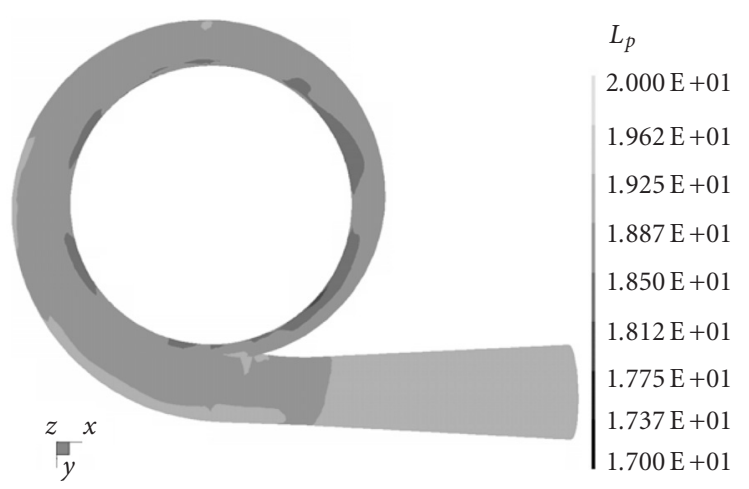

(c)

FIGURE 22: Static pressure on the volute surface for the vaned case at near-surge conditions: (a) starting position, (b) $1 / 3$ revolution, and (c) $2 / 3$ revolution.

compressor characteristics of performance, vibration, and acoustics.

The evolution of the standing waves near the diffuser vanes reveals that matching the numbers of impeller blades and diffuser vanes impacts the dynamic feature of the flow field. Although more detailed work needs to be done to provide a better understanding of the mechanism controlling the 
formation and evolution of these standing waves, the conventional design practice to avoid common divisors has its physical foundation.

\section{ACKNOWLEDGMENTS}

The author would like to thank TRANE Air Conditioning, American Standard Companies, for the permission to publish this paper. Jack Sauls of TRANE Air Conditioning, American Standard Companies, reviewed the manuscript.

\section{REFERENCES}

[1] Y. Yoshinaga, I. Gyobu, H. Mishina, F. Koseki, and H. Nishida, "Aerodynamic performance of a centrifugal compressor with vaned diffusres," ASME Journal of Fluid Enginering, vol. 102, pp. 486-493, 1980.

[2] M. B. Flathers, "Design and retrofit of a low solidity diffuser for a pipeline centrifugal compressor application," in Proc. 26th Turbomachinery Symposium, pp. 57-66, Houston, Tex, USA, 1997.

[3] T. Kmecl and P. Dalbert, "Investigation of the Influence of Geometry Parameters on Performance of a Diffuser," 1999, ASME Paper No. 99-GT-437.

[4] M. M. Cui, "Comparative study of vaneless and vaned diffusers in a transonic centrifugal compressor with real gas," 2000, ASME Paper 2000-GT-0450.

[5] Y. Senoo, H. Hayami, and H. Ueki, "Low-solidity tandemcascade diffusers for wide-flow-range centrifugal blowers," 1983, ASME Paper No. 83-GT-3.

[6] J. S. Kang and S. H. Kang, "Unsteady Flow Phenomena in a Centrifugal Compressor Channel Diffuser," 2000, ASME Paper No. 2000-GT-451.

[7] D. Hagelstein, R. Van den Brambussche, R. Keiper, and M. Rautenberg, "Experimental Investigation of the Circumferential Static Pressure Distribution in Centrifugal Compressor Stages," 1997, ASME Paper No. 97-GT-50.

[8] C. Roduner, P. Kupferschmied, P. Koppel, and G. Gyarmathy, "On the development and application of the fast-response aerodynamic probe system in turbomachines-part 2: flow, surge, and stall in a centrifugal compressor," Journal of Turbomachinery, vol. 122, pp. 517-526, 2000. 

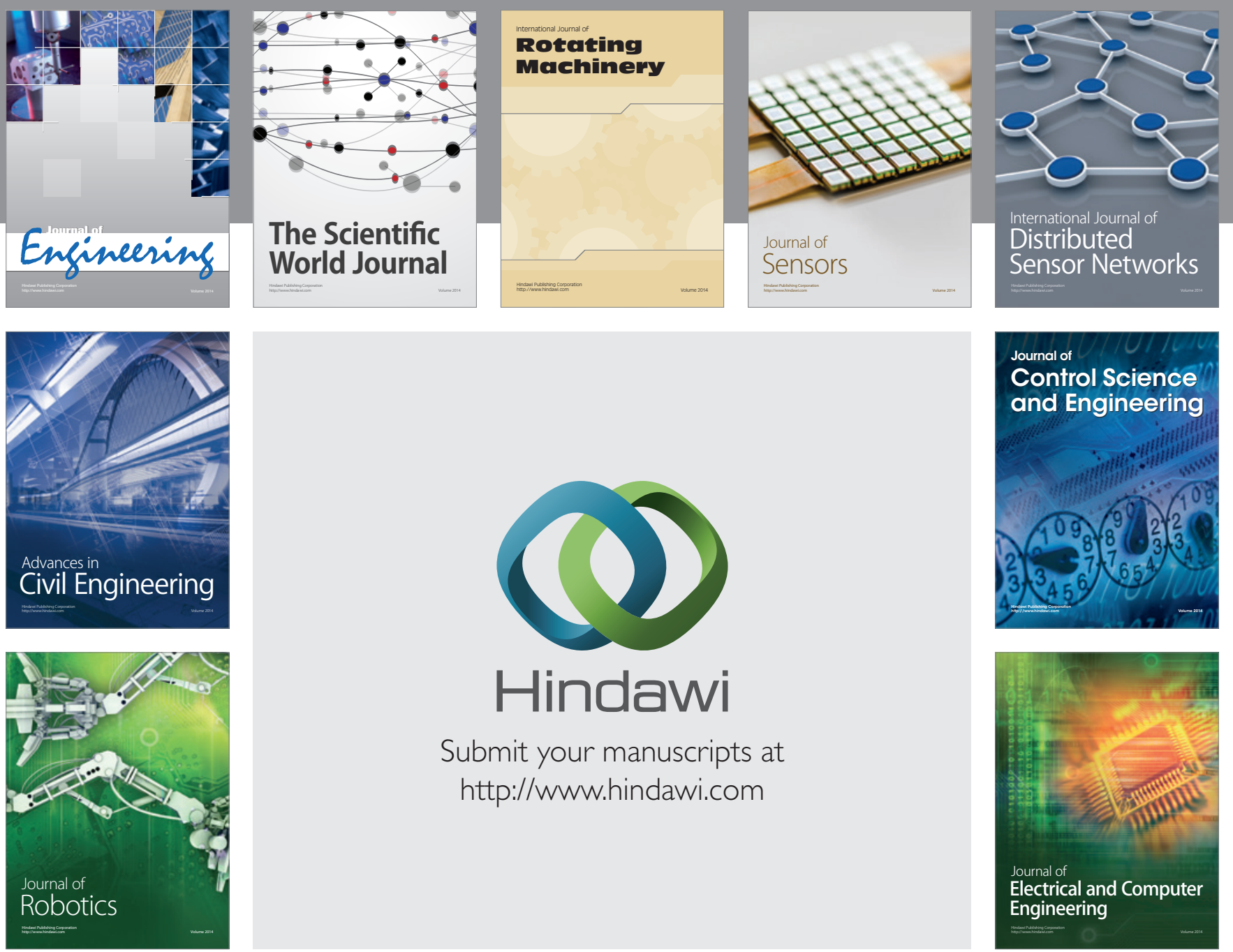

Submit your manuscripts at

http://www.hindawi.com
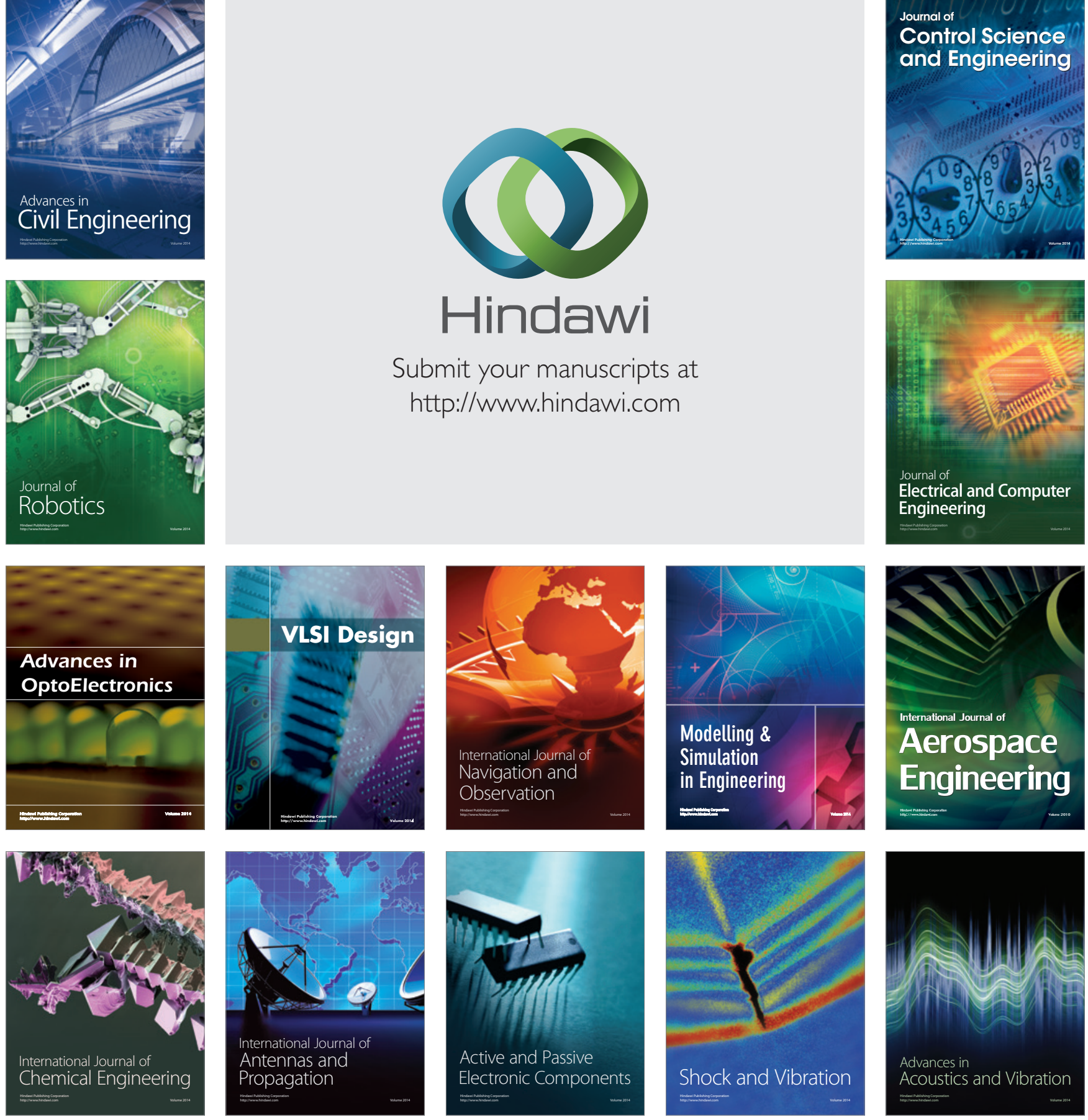